\title{
»Haben Sie noch einen Wunsch aus der Mini-Bar?«
}

\author{
Joachim Vetter
}

Das Pflegeversicherungsgesetz eröffnet für die Leistungsanbieter die Möglichkeit, Zuschläge für Zusatzleistungen anzubieten. Diese Zuschläge müssen allerdings zwischen dem Pflegeheim und dem Bewohner gesondert und schriftlich vereinbart werden.

Mit dem üblichen Heimentgelt werden die Leistungen abgegolten, die das Heim als Regelleistung anbietet. Jeder Heimträger ist gesetzlich verpflichtet, diese Leistungen nach Art und Umfang im Einzelnen zu benennen. Diese Regelleistungen werden seit einigen Jahren in den Leistungs- und Qualitätsvereinbarungen festgelegt und werden für Kostenträger und Kunden nachvollziehbar.

In Heimverträgen mit Versicherten der sozialen Pflegeversicherung (nicht mit einer privaten Pflegeversicherung angehörenden Personen), die pflegebedürftig sind und Leistungen der stationären Pflege in Anspruch nehmen, müssen die Leistungen für allgemeine Pflegeleistungen, für Unterkunft und Verpflegung sowie für Zusatzleistungen besonders beschrieben werden und die Entgelte hierfür im einzelnen angegeben werden. Für Preisvergleiche müssen also die Leistungsangebote gegenübergestellt werden. Innerhalb der Heime sind die Leistungen in der Regel für Kunden und Kostenträger vergleichbar.

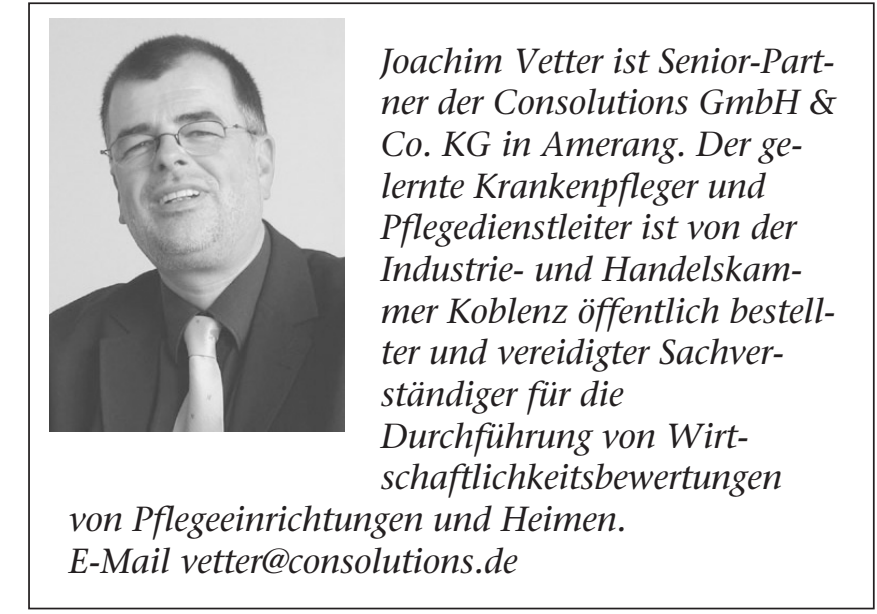

\section{Marktüberblick}

Immer mehr stationäre Einrichtungen bieten zusätzlich zu den mit dem Heimentgelt abgegoltenen Leistungen Sonderleistungen an, die dann auch gesondert bezahlt werden müssen. Die Art und die Kosten dieser Sonderoder Zusatzleistungen sind meist in einer Liste aufgeführt, die den Bewohnern zugänglich sein muss (vgl. Kasten). $\rightarrow$

\section{Zusatzleistungen in der Pflegeversicherung}

§ 88 Zusatzleistungen

(1) Neben den Pflegesätzen nach § 85 und den Entgelten nach § 87 darf das Pflegeheim mit den Pflegebedürftigen über die im Versorgungsvertrag vereinbarten notwendigen Leistungen hinaus ( 72 Abs. 1 Satz 2 ) gesondert ausgewiesene Zuschläge für

- besondere Komfortleistungen bei Unterkunft und Verpflegung sowie

- zusätzliche pflegerisch-betreuende Leistungen

vereinbaren (Zusatzleistungen). Der Inhalt der notwendigen Leistungen und deren Abgrenzung von den Zusatzleistungen werden in den Rahmenverträgen nach $§ 75$ festgelegt.

(2) Die Gewährung und Berechnung von Zusatzleistungen ist nur zulässig, wenn:

- dadurch die notwendigen stationären oder teilstationären Leistungen des Pflegeheimes (§ 84 Abs. 4 und § 87) nicht beeinträchtigt werden,

- die angebotenen Zusatzleistungen nach Art, Umfang, Dauer und Zeitabfolge sowie die Höhe der Zuschläge und die Zahlungsbedingungen vorher schriftlich zwischen dem Pflegeheim und dem Pflegebedürftigen vereinbart worden sind,

- das Leistungsangebot und die Leistungsbedingungen den Landesverbänden der Pflegekassen und den überörtlichen Trägern der Sozialhilfe im Land vor Leistungsbeginn schriftlich mitgeteilt worden sind.

Sozialgesetzbuch XI - Soziale Pflegeversicherung 
Immer wieder ausgehandelte Zusatzleistungen sind beispielsweise die nachfolgenden Angebote:

- Reisebegleitung beispielsweise zum Grab des verstorbenen Ehegatten; Berechnung nach Aufwand

- Telefonanschlussgebühren und Gesprächseinheiten, so es sich nicht um einen Amtsanschluss der Telekom handelt und die Gebühren direkt mit den Bewohnern abgerechnet werden

- Aufwendungen für persönliche Bedürfnisse nach Aufwand, beispielsweise Prosecco zum Frühstück oder die Versorgung eines Kanarienvogels

- ärztliche Betreuung: Die Kosten für die ärztliche Betreuung werden den Bewohnerinnen und Bewohnern durch den behandelnden Arzt direkt in Rechnung gestellt

- Todesfall: Das Heimentgelt wird bis zum Todestag erhoben. Für die Aufwendungen des Altenpflegeheimes im Zusammenhang mit der Räumung, Entsorgung, Sanierung und Wiederbelegung des Zimmers kann eine Aufwandpauschale in Rechnung gestellt werden.

- Bargeldverwaltung: Einige Heime verwalten das Bargeld der Bewohner im Auftrag der Bewohner selbst oder deren gesetzlichen Betreuer. Die dem Heim damit entstehenden Aufwendungen können mit einer Kontoverwaltungspauschale je Monat sichergestellt werden. Diese betragen zwischen vier und zehn Euro monatlich.
- Einzelzimmerzuschlag im Pflegeheim, allerdings unter Beachtung eines Urteils des Bundesgerichtshofes (vgl. Kasten)

Zusatzleistungen für Einrichtungen sollten individuell entwickelt, bewohner- und bedürfnisorientiert angeboten werden. Diese Zusatzleistungen müssen den Kostenträgern angezeigt werden. Beispiel: Die Pflegeeinrichtung installiert zur Förderung des Wohlbefindens der Bewohner ein Sanarium und stellt dieses gegen Gebühr zur Nutzung zur Verfügung. (Dabei handelt es sich um eine kreislaufschonende Saunavariante, bei der die Raumtemperatur zwischen 46 und 60 Celsius und die Luftfeuchtigkeit zwischen 40 und 55 Prozent liegt.) Das Sanarium kann nach ärztlicher Empfehlung von Bewohnern in Anspruch genommen werden. Die Nutzung wird mit zwölf Euro je 60 Minuten berechnet. Ähnliche Regelungen sind für Luftwäscher in Bewohnerzimmern bei passionierten Rauchern denkbar. Alle Zusatzleistungen müssen in jedem Falle im Heimvertrag unter Zusatzleistungen definiert und der Preis für diese Leistungen explizit bekannt sein.

Mehr und mehr werden Kioskleistungen, die nicht als Zusatzleistungen im herkömmlichen Sinne bezeichnet werden müssen, in Pflegeheimen üblich. Die mobile Minibar am Abend bietet vom süßen Snack über ein kleines Eis auch Bier und das Glas Rotwein an. Diese Leistungen werden sofort vom Bewohner gezahlt. Entscheidend ist hierbei die Abstimmung mit Betreuern, wenn Bewohner selbst nicht geschäftsfähig sind und solche kleine Zusatzleistungen beispielsweise durch Nachtwachen angeboten werden.

\section{Bundesgerichtshof: Einzelzimmerzuschlag in Pflegeheim}

Die Parteien streiten im Revisionsverfahren noch über die Frage, ob die Beklagte - Trägerin eines Alten- und Pflegeheims für die Bereitstellung eines Einzelzimmers einen Zuschlag berechnen darf. Die frühere Klägerin, die im Laufe des Rechtsstreits verstorben ist, wurde auf der Grundlage eines »Heim-Vorvertrags« vom 27.8.1997 am 10.9.1997 in das Pflegeheim aufgenommen.

Sie erhielt Leistungen der Pflegeversicherung nach Pflegestufe III und wurde über eine PEG-Sonde künstlich ernährt. Sie bewohnte von Beginn an als Einzelperson ein Zimmer, das der Größe nach auf eine Belegung durch zwei Personen zugeschnitten war. Der geschlossene Vertrag sah über die Inanspruchnahme eines solchen Zimmers und die hierfür zu entrichtende Vergütung nichts vor. Nach dem Vorvertrag war zwar der Abschluss eines endgültigen Wohn- und Dienstleistungsvertrags nach Veröffentlichung eines erst noch zu schließenden Rahmenvertrags nach § 75 SGB XI vorbehalten, zu einem solchen Vertragsschluss kam es aber nicht.

$\mathrm{Ab}$ dem 1.1.1998 berechnete die Beklagte einen täglichen Einzelzimmerzuschlag von 57,90 DM, später 29,60 EUR, der durch den Betreuer der Klägerin, der sie nach ihrem Tod auch beerbt hat, bis zum 31.1.2003 bezahlt wurde. Gegenstand der Klage ist die Rückzahlung der gezahlten Einzelzimmerzuschläge, die der Kläger mit der Begründung verlangt, nach § 88 Abs. 2 Nr. 2 SBG XI sei die Gewährung und Berechnung von Zusatzleistungen nur zulässig, wenn die angebotenen Zusatzleistungen nach Art, Umfang, Dauer und Zeitfolge sowie die Höhe der Zuschläge und die Zahlungsbedingungen vorher schriftlich zwischen dem Pflegeheim und den Pflegebedürftigen vereinbart worden seien; mit ihrer Widerklage verfolgt die Beklagte die Zahlung von Einzelzimmerzuschlägen vom 1.2.2003 bis 31.12.2003.

Das Landgericht hat dem Kläger Recht gegeben und ihm insoweit 54.972,75 EUR zugesprochen. Das Berufungsgericht hat eine Rückforderung nur in Höhe von 25.260,75 EUR für gerechtfertigt gehalten und den Kläger auf die Widerklage zur Zahlung von $5.437,74$ EUR verurteilt. Es ist zwar auch davon ausgegangen, dass es an einer wirksamen Vereinbarung über den Einzelzimmerzuschlag fehle. Es hat aber gemeint, der Beklagten stehe wegen der Inanspruchnahme des Einzelzimmers ein Bereicherungsanspruch in Höhe von 16,00 EUR täglich zu.

Der III. Zivilsenat hat das landgerichtliche Urteil wiederhergestellt, soweit es um die Einzelzimmerzuschläge geht. Er hat wie beide Vorinstanzen entschieden, dass es zur Gewährung und Berechnung von Zusatzleistungen in Heimverträgen mit Leistungsempfängern der Pflegeversicherung einer vorherigen schriftlichen Vereinbarung zwischen dem Pflegebedürftigen und dem Heimträger bedarf.

Um dem Schutzinteresse des Pflegebedürftigen zu genügen, dem der Formzwang in § 88 Abs. 2 Nr. 2 SGB XI dient, hat der III. Zivilsenat in Anlehnung an seine Rechtsprechung über unwirksame Wahlleistungsvereinbarungen nach der Bundespflegesatzverordnung auch Bereicherungsansprüche wegen der Nutzung solcher Zusatzleistungen abgelehnt.

Dies schließt im Einzelfall zwar nicht aus, dass es einem Heimbewohner nach dem Grundsatz von Treu und Glauben versagt sein kann, sich auf den Formmangel einer Vereinbarung zu berufen. Das ist aber grundsätzlich nur bei einem grob treuwidrigen Verhalten anzunehmen, das der Senat im Streitfall verneint hat. Grundsätzlich kann von einem Heimträger, der eine Vielzahl von Heimverträgen formularmäßig abschließt, erwartet werden, dass er auf den Abschluss einer schriftlichen Vereinbarung achtet, ehe er gesondert berechenbare Zusatzleistungen gewährt.

Urteil vom 13. Oktober 2005 - III ZR 400/04 LG Nürnberg-Fürth; Entscheidung vom 27.2.2004 - 13 O 3886/03 ./. OLG Nürnberg; Karlsruhe, den 13. Oktober 2005 